\title{
HMG-CoA reductase expression in primary colorectal cancer correlates with favourable clinicopathological characteristics and an improved clinical outcome
}

Erik Bengtsson ${ }^{1 \dagger}$, Pashtrik Nerjovaj $^{1 \dagger}$, Sakarias Wangefjord ${ }^{1}$, Björn Nodin ${ }^{1}$, Jakob Eberhard ${ }^{1}$, Mathias Uhlén ${ }^{2,3}$, Signe Borgquist ${ }^{1}$ and Karin Jirström ${ }^{1 *}$

\begin{abstract}
Background: An association between tumor-specific HMG-CoA reductase (HMGCR) expression and good prognosis has previously been demonstrated in breast and ovarian cancer. In this study, the expression, clinicopathological correlates and prognostic value of HMGCR expression in colorectal cancer was examined.

Findings: Immunohistochemical expression of HMGCR was assessed in tissue microarrays with primary tumours from 557 incident cases of colorectal cancer in the Malmö Diet and Cancer Study. Pearson's Chi Square test was applied to explore the associations between HMGCR expression and clinicopathological factors and other investigative biomarkers. Kaplan Meier analysis and Cox proportional hazards modeling were used to assess the relationship between HMGCR expression and cancer-specific survival (CSS) according to negative vs positive HMGCR expression.

A total number of 535 (96.0\%) tumours were suitable for analysis, of which 61 (11.4\%) were HMGCR negative. Positive cytoplasmic HMGCR expression was associated with distant metastasis-free disease at diagnosis $(p=0.002)$, lack of vascular invasion ( $p=0.043)$, microsatellite-instability $(p=0.033)$, expression of cyclin D1 $(p=<0.001)$ and p21 ( $p=<0.001$ ). Positive HMGCR expression was significantly associated with a prolonged CSS in unadjusted Cox regression analysis in the entire cohort ( $\mathrm{HR}=1.79 ; 95 \% \mathrm{Cl} 1.20-2.66)$ and in Stage III-IV disease $(H R=1.71 ; 95 \%$ Cl 1.09-2.68), but not after adjustment for established clinicopathological parameters.

Conclusions: Findings from this prospective cohort study demonstrate that HMGCR is differentially expressed in colorectal cancer and that positive expression is associated with favourable tumour characteristics and a prolonged survival in unadjusted analysis. The utility of HMGCR as a predictor of response to neoadjuvant or adjuvant statin treatment in colorectal cancer merits further study.
\end{abstract}

Virtual slides: The virtual slides for this article can be found here: http://www.diagnosticpathology.diagnomx.eu/vs/ 2115647072103464.

Keywords: HMG-CoA reductase, Immunohistochemistry, Colorectal cancer, Prognosis

\footnotetext{
* Correspondence: karin.jirstrom@med.lu.se

${ }^{\dagger}$ Equal contributors

'Department of Clinical Sciences, Oncology and Pathology, Lund University,

22185 Lund, Sweden

Full list of author information is available at the end of the article
} 


\section{Findings}

\section{Background}

Colorectal cancer (CRC) is the third most common form of cancer in the world, with an estimated annual global incidence of more than 1.2 million new cases and around 600000 deaths from the disease [1]. Although many efforts have been made to find molecular markers to identify high-risk disease and to select patients for adjuvant treatment, none have yet been implemented into routine clinical practice.

It has recently been demonstrated that elevated expression of the enzyme 3-hydroxy-3-methylglutharyl-coenzyme A reductase (HMGCR) correlates with favourable prognosis in breast and ovarian cancer [2,3], and with an improved response to tamoxifen [4] and pre-surgical statin treatment [5] in breast cancer. Several epidemiological studies have shown a link between use of statins, inhibitors of HMGCR, and a significantly reduced CRC incidence [6-8], and statins have also been demonstrated to exert anti-neoplastic properties in CRC cells in vitro $[9,10]$.

To the best of our knowledge, the expression and prognostic significance of HMGCR in CRC has not yet been reported. The aim of this study was therefore to analyse tumour-specific expression of HMGCR by immunohistochemistry in tissue microarrays (TMAs) with primary tumour specimens from incident CRC cases diagnosed within a large, prospective cohort study, and to explore its relationship with established clinicopathological and tumor biological parameters, and survival.

\section{Methods}

Until 31 Dec 2008, 626 incident cases of CRC had been registered in the Malmö Diet and Cancer Study (MDCS) [11-13]. Cases were identified from the Swedish Cancer Registry up until 31 Dec 2007, and from The Southern Swedish Regional Tumour Registry for the period of 1 Jan - 31 Dec 2008. Information on vital status and cause of death was obtained from the Swedish Cause of Death Registry up until 31 Dec 2009. Follow-up started at date of diagnosis and ended at the date of death, emigration or end of follow-up, whichever came first. All tumours with available slides or paraffin blocks were histopathologically re-evaluated on haematoxylin and eosin stained slides. Histopathological, clinical and treatment data were obtained from the clinical and/or pathology records. TNM staging was performed according to the American Joint Committee on Cancer (AJCC) [14]. Patient and tumour characteristics of the cohort have been described in detail previously [15-19]. Ethical permission was obtained from the Ethics Committee at Lund University (Ref. nr 51/90 and 530/2008).

TMAs were constructed as previously described using duplicate $1.00 \mathrm{~mm}$ cores [16].
For immunohistochemical analysis of HMGCR, $4 \mu \mathrm{m}$ TMA-sections were automatically pre-treated using the PT-link system (DAKO, Glostrup, Denmark) and then stained in an Autostainer Plus (DAKO) with a polyclonal anti-HMGCR antibody HPA008338, Atlas Antibodies AB, Stockholm, Sweden diluted 1:150. This antibody has been validated (www.proteinatlas.org) and used in a recent window-of-opportunity statin trial in breast cancer [5].

HMGCR was expressed in the cytoplasm of the tumour cells, without distinct membranous staining as previously shown in e.g. breast cancer [2]. In line with previous studies, HMGCR was generally expressed in $>50$ percent of tumour cells in all positive cases $[2,3,20]$. Therefore, only the cytoplasmic staining intensity was taken into account, and denoted as $0=>1 \%$ of cells being negative, $1=$ weak expression in any fraction $>1 \%$, and $2=$ moderate/strong expression in any fraction $>1 \%$. The staining was evaluated by three independent observers, who were blinded to clinical and outcome data $(\mathrm{EB}, \mathrm{PN}$ and $\mathrm{KJ})$. Scoring differences were discussed in order to reach consensus.

Immunohistochemical staining and evaluation of cyclin D1, p21, p27, p53, and beta-catenin expression has been described previously [15,17]. Immunohistochemical staining for microsatellite instability (MSI) screening status was performed as previously described, whereby tumours lacking expression of any of the DNA mismatch repair proteins MLH1, PMS2, MSH2 or MSH6 were denoted as having a positive MSI screening status, and tumours expressing all MMR proteins as being microsatellite stable (MSS) [18]. Analysis of KRAS and BRAF mutational status was performed by pyrosequencing as previously described [21,22]. Pearson's Chi Square test was used to explore the associations of HMGCR expression with clinicopathological and investigative parameters. Kaplan-Meier analysis and log rank test were used to illustrate differences in cancer specific survival (CSS) according to negative vs positive HMGCR expression. Cox regression proportional hazards models were used for estimation of hazard ratios (HRs) for death from CRC and overall death in both uniand multivariable analysis, the latter adjusted for age, sex, tumour $(\mathrm{T})$-stage, lymph node metastasis $(\mathrm{N}$-stage), distant metastasis (M-stage), differentiation grade, vascular invasion and MSI status. A backward conditional selection method was used for variable selection by the model. All tests were two-sided. A p-value of 0.05 was considered significant. All statistical analyses were performed using IBM SPSS Statistics version 20.0.

\section{Results}

HMGCR expression could be evaluated in 535 (96.0\%) cases, of which 61 (11.4\%) were negative, 206 (38.5\%) displayed weak and 268 (50.1\%) displayed strong expression. 
Representative immunohistochemical images are shown in Figure 1. Normal colonic mucosa displays moderate-strong HMGCR expression (www.proteinatlas.org).

As shown in Table 1, there were significant associations between HMGCR expression and distant metastasis-free disease $(\mathrm{p}=0.002)$, lack of vascular invasion $(\mathrm{p}=0.043)$ and positive MSI screening status $(\mathrm{p}=0.033)$. Moreover,
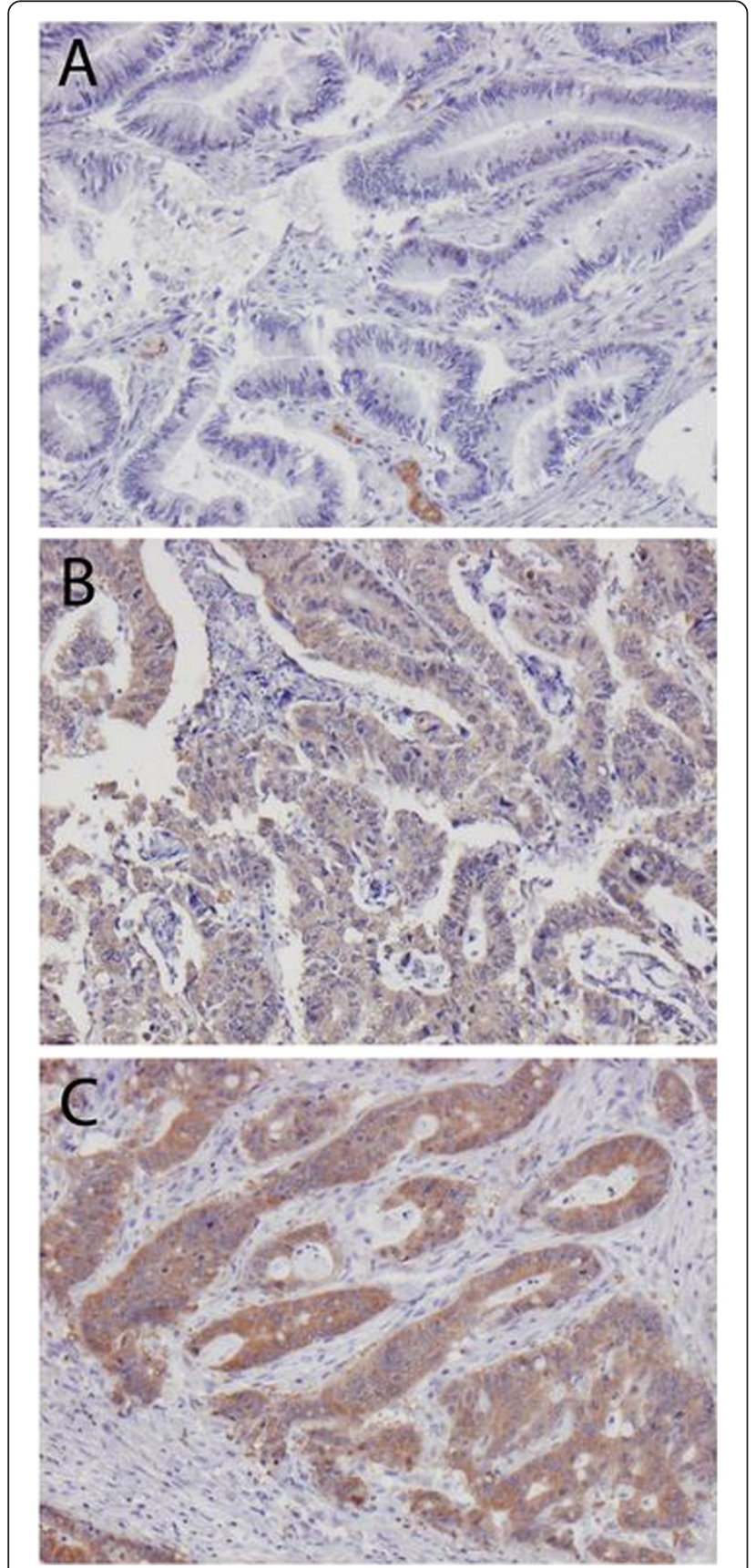

Figure $1 \mathrm{HMG}-\mathrm{Co} A$ reductase expression in colorectal cancer. Sample immuohistochemical images (10X) of primary colorectal cancer with (A) negative; (B) weak and (C) strong HMG-CoA reductase expression.
Table 1 Significant associations of HMG-CoA reductase expression with clinicopathological characteristics and investigative factors

\begin{tabular}{|c|c|c|c|}
\hline \multirow[b]{2}{*}{ Factor } & \multicolumn{2}{|c|}{ HMG-CoA reductase expression } & \multirow{3}{*}{ P-value } \\
\hline & Negative & Positive & \\
\hline$N(\%)$ & $61(11.4)$ & $474(88.6)$ & \\
\hline \multicolumn{4}{|l|}{ M stage } \\
\hline 0 & $41(68.3)$ & $395(84.4)$ & 0.002 \\
\hline 1 & $19(31.7)$ & $73(15.6)$ & \\
\hline Missing & 1 & 6 & \\
\hline \multicolumn{4}{|c|}{ Vascular invasion } \\
\hline No & $11(32.4)$ & $142(50.7)$ & 0.043 \\
\hline Yes & $23(67.6)$ & $138(49.3)$ & \\
\hline Missing & 17 & 194 & \\
\hline \multicolumn{4}{|c|}{ Microsatellite instability } \\
\hline MSS & $52(94.5)$ & $371(83.6)$ & 0.033 \\
\hline MSI & $3(5.5)$ & $73(16.4)$ & \\
\hline Missing & 6 & 30 & \\
\hline \multicolumn{4}{|c|}{ Cyclin D1 expression } \\
\hline Negative & $22(37.3)$ & $80(17.7)$ & $<0.001$ \\
\hline Positive & $37(62.7)$ & $371(82.3)$ & \\
\hline Missing & 2 & 23 & \\
\hline \multicolumn{4}{|c|}{ p21 expression } \\
\hline Negative & $20(33.9)$ & $53(11.8)$ & $<0.001$ \\
\hline Positive & $39(66.1)$ & $396(88.2)$ & \\
\hline missing & 2 & 25 & \\
\hline
\end{tabular}

The categories marked as missing were not included in the analysis.

HMGCR expression was significantly associated with expression of cyclin D1 $(\mathrm{p}<0.001)$ and $\mathrm{p} 21(\mathrm{p}<0.001)$. There were no significant associations between HMGCR expression and age, sex, T-stage, N-stage, differentiation grade, mucinous histology, beta-catenin grades, p53 status, KRAS or BRAF mutation (data not shown).

Kaplan-Meier analysis and log rank test revealed a significant association between positive HMGCR expression and a prolonged CSS in the full cohort and in Stage III-IV (metastatic) disease, but not in Stage I-II (nonmetastatic) disease (Figure 2). Notably, survival rates were similar for tumours with weak and moderate/strong expression (Figure 2).

Unadjusted Cox regression analysis confirmed the significant correlation between positive HMGCR expression and an improved CSS in the entire cohort ( $\mathrm{HR}=1.79 ; 95 \%$ CI 1.20-2.66) and in stage III-IV disease (HR $=1.71$ (1.092.68) (Table 2). However, these associations did not remain significant in multivariable Cox regression analysis, adjusted for age, sex, T-stage, $\mathrm{N}$-stage, M-stage, differentiation grade, vascular invasion and MSI status (Table 2). Similar associations were found using overall survival as 

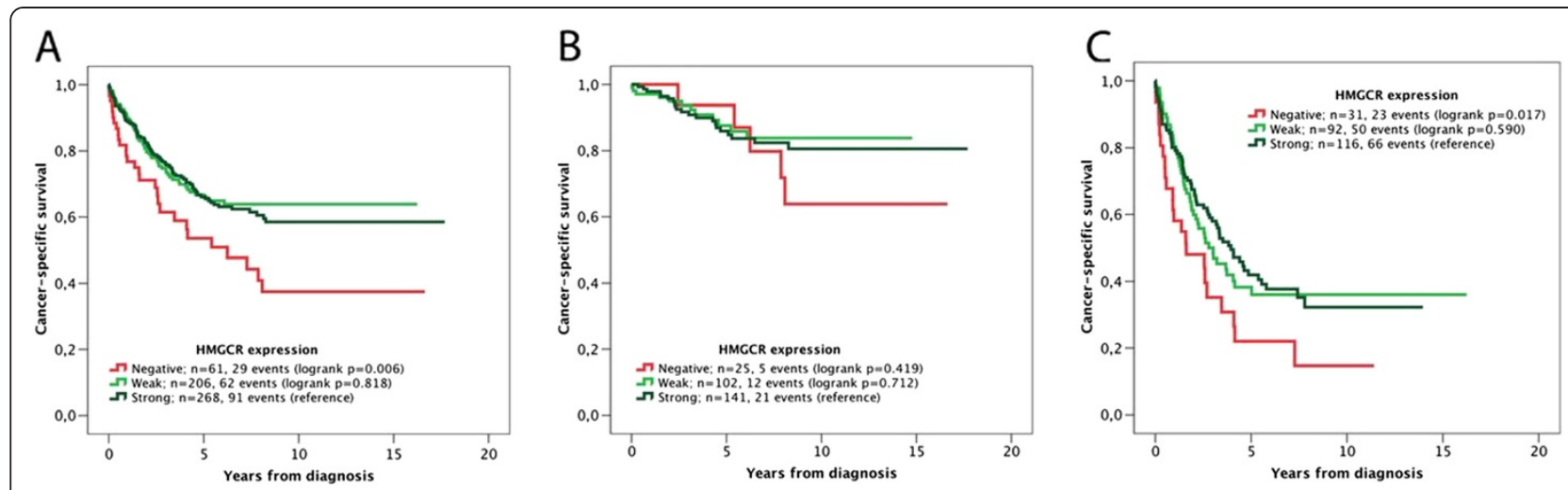

Figure 2 Kaplan-Meier estimates of the prognostic impact of HMG-CoA reductase expression. Kaplan Meier analysis and log rank test of colorectal cancer specific survival according to negative, weak and strong HMGCR expression in (A) all patients, (B) patients with stage I-II disease and (C) patients with stage III-IV disease.

endpoint (data not shown). The prognostic value of HMGCR did not differ by sex or tumour location (data not shown). There was no significant association between HMGCR expression and response to adjuvant treatment in curatively treated patients with stage III-IV disease (data not shown).

\section{Discussion}

The results from this comparatively large cohort study demonstrate that HMGCR expression in CRC is associated with clinically less advanced tumours and a more favourable prognosis. These findings are in line with previous studies on e.g. breast and ovarian cancer $[2,3]$.

The correlation between HMGCR expression and a prolonged CSS did however not remain significant in adjusted analysis, and the lack of independent prognostic information for HMGCR expression may be explained by its significant association with established prognostic factors, in particular M-stage and vascular invasion. As the results in this study are entirely based on analysis of primary tumours, it would also be of interest to compare HMGCR expression in primary and metastatic lesions in future studies [23,24]. Moreover, as data on recurrent disease was not available for the patients in this cohort, future studies should also consider the association of HMGCR expression with to time to recurrence, preferably in cohorts where this information has been recorded prospectively.

The finding of an association between HMGCR expression and positive MSI screening status is of potential interest, as MSI tumours may be less responsive to adjuvant treatment with 5-fluorouracil-based chemotherapy [25-27]. Speculatively, CRC patients with MSI/HMCGR expressing tumours may instead benefit from adjuvant statin treatment, in particular as HMGCR expression has been demonstrated to predict an improved response to pre-surgical statin treatment in breast cancer [5]. Notably, in this cohort, positive MSI status has been demonstrated to be associated with a significantly improved prognosis in patients with stage III-IV disease, but not to be predictive of response to adjuvant chemotherapy [15]. Future studies should also address whether HMGCR expression in CRC is affected by different neoadjuvant treatment regimens $[24,28]$.

Unfortunately, it was not possible to examine associations of HMGCR expression with statin use in this cohort,

Table 2 Cox proportional hazards analysis of colorectal cancer specific survival according to HMG-CoA reductase expression

\begin{tabular}{|c|c|c|c|c|c|c|}
\hline & \multicolumn{2}{|c|}{ Full cohort } & \multicolumn{2}{|c|}{ Stage I-II disease } & \multicolumn{2}{|c|}{ Stage I-II disease } \\
\hline & HR $(95 \% \mathrm{Cl})$ & $n$ (events) & HR $(95 \% \mathrm{Cl})$ & $n$ (events) & HR $(95 \% \mathrm{Cl})$ & $\overline{n \text { (events) }}$ \\
\hline & \multicolumn{2}{|c|}{ Unadjusted } & \multicolumn{2}{|c|}{ Unadjusted } & \multicolumn{2}{|c|}{ Unadjusted } \\
\hline HMGCR pos & 1.00 & $474(153)$ & 1,00 & $243(33)$ & 1,00 & $208(116)$ \\
\hline \multirow[t]{2}{*}{ HMGCR neg } & $1.79(1-20-2.66)$ & $61(29)$ & $1.56(0.61-4.01)$ & $25(5)$ & $1.71(1.09-2.68)$ & $31(23)$ \\
\hline & \multicolumn{2}{|c|}{ Adjusted } & \multicolumn{2}{|c|}{ Adjusted } & \multicolumn{2}{|c|}{ Adjusted } \\
\hline HMGCR pos & 1.00 & $406(126)$ & 1.00 & $227(30)$ & 1.00 & $179(96)$ \\
\hline HMGCR neg & $0.85(0.51-1.41)$ & $45(19)$ & $1.23(0.41-3.70)$ & $23(4)$ & $0.82(0.46-1.46)$ & $22(15)$ \\
\hline
\end{tabular}

Multivariable analysis included adjustment for age (continuous), T-stage (1-2, 3, 4), sex, N-stage (0,1,2), M-stage, differentiation grade (high-intermediate vs. low), vascular invasion (no, yes, unknown) and MSI screening status. 
since the number of cases for whom statin medication was reported at baseline was too small [2]. However, a previous study demonstrated associations of high tumour-specific HMGCR expression in post-menopausal breast cancer with use of hormone replacement therapy and obesity [2]. These findings suggest that the influence of lifestyle factors and anthropometric measures on HMGCR expression in CRC also merits further study.

Statin-mediated blockade of the mevalonate pathway triggers a marked increase of inactive HMGCR in cultured cells [29] and statin treatment has also been demonstrated to induce cell cycle arrest and up-regulation of p21 and p27 in colorectal cancer in vitro [10]. In this context, the significant associations of HMGCR expression with expression of p21 and cyclin D1found here also indicate a potential cell-cycle regulatory role for HMGCR in colorectal cancer in vivo. Of note, high cyclin D1 expression has previously been found to correlate with good prognosis in this cohort, in particular in male CRC [17].

\section{Conclusions}

This study provides a first report of tumour-specific HMGCR in colorectal cancer, and its association with favourable clinicopathological characteristics and an improved prognosis. The utility of HMGCR expression as a predictor of response to different neoadjuvant or adjuvant treatment regimens in colorectal cancer should be investigated in future studies.

\section{Abbreviations}

CRC: Colorectal cancer; CSS: Cancer specific survival; HMGCR: 3-hydroxy3-methylglutharylcoenzyme A reductase; HR: Hazard ratio; IHC: Immunohistochemistry; MDCS: Malmö Diet and Cancer Study; MSI: Microsatellite instability; MSS: Microsatellite stability; TMA: Tissue microarray.

\section{Competing interests}

The authors declare that no competing interests exist.

\section{Authors' contributions}

EB and PN performed the immunohistochemical evaluation, statistical analyses and drafted the manuscript. BN carried out the $\mathrm{IHC}$ stainings and assisted with the collection of clinical data. SW and JE collected clinical, follow-up and treatment data. MU contributed to the conception and design of the study. SB conceived of the study and helped draft the manuscript. KJ conceived of the study, carried out the histopathological re-evaluation, evaluated the immunohistochemistry, and helped drafted the manuscript. All authors read and approved the final manuscript.

\section{Acknowledgements}

This study was supported by grants from the Knut and Alice Wallenberg Foundation, the Swedish Cancer Society, the Gunnar Nilsson Cancer Foundation, the Swedish Government Grant for Clinical Research, Lund University Faculty of Medicine and University Hospital Research Grants.

\section{Author details}

${ }^{1}$ Department of Clinical Sciences, Oncology and Pathology, Lund University, 22185 Lund, Sweden. ${ }^{2}$ Science for Life Laboratory, Royal Institute of Technology, 17121 Stockholm, Sweden. ${ }^{3}$ School of Biotechnology, AlbaNova University Center, Royal Institute of Technology, 10691 Stockholm, Sweden.
Received: 22 July 2013 Accepted: 29 March 2014

Published: 7 April 2014

\section{References}

1. Ferlay J, Shin HR, Bray F, Forman D, Mathers C, Parkin DM: Estimates of worldwide burden of cancer in 2008: GLOBOCAN 2008. Int J Cancer J Int Du Cancer 2010, 127(12):2893-2917.

2. Borgquist S, Djerbi S, Ponten F, Anagnostaki L, Goldman M, Gaber A, Manjer J, Landberg G, Jirstrom K: HMG-CoA reductase expression in breast cancer is associated with a less aggressive phenotype and influenced by anthropometric factors. Int J Cancer 2008, 123(5):1146-1153.

3. Brennan DJ, Brandstedt J, Rexhepaj E, Foley M, Ponten F, Uhlen M, Gallagher WM, O'Connor DP, O'Herlihy C, Jirstrom K: Tumour-specific HMG-CoAR is an independent predictor of recurrence free survival in epithelial ovarian cancer. BMC Cancer 2010, 10:125.

4. Brennan DJ, Laursen H, O'Connor DP, Borgquist S, Uhlen M, Gallagher WM, Ponten F, Millikan RC, Ryden L, Jirstrom K: Tumor-specific HMG-CoA reductase expression in primary premenopausal breast cancer predicts response to tamoxifen. Breast Cancer Res 2011, 13(1):R12.

5. Bjarnadottir $\mathrm{O}$, Romero $\mathrm{Q}$, Bendahl PO, Jirstrom K, Ryden L, Loman N, Uhlen M, Johannesson H, Rose C, Grabau D, Borgquist S: Targeting HMG-CoA reductase with statins in a window-of-opportunity breast cancer trial. Breast Cancer Res Treat 2013, 138(2):499-508.

6. Broughton T, Sington J, Beales IL: Statin use is associated with a reduced incidence of colorectal cancer: a colonoscopy-controlled case-control study. BMC Gastroenterol 2012, 12:36.

7. Poynter JN, Gruber SB, Higgins PD, Almog R, Bonner JD, Rennert HS, Low M, Greenson JK, Rennert G: Statins and the risk of colorectal cancer. New Engl J Med 2005, 352(21):2184-2192.

8. Lipkin SM, Chao EC, Moreno V, Rozek LS, Rennert H, Pinchev M, Dizon D, Rennert G, Kopelovich L, Gruber SB: Genetic variation in 3-hydroxy-3methylglutaryl CoA reductase modifies the chemopreventive activity of statins for colorectal cancer. Cancer Prev Res (Phila) 2010, 3(5):597-603.

9. Feleszko W, Jakobisiak M: Lovastatin augments apoptosis induced by chemotherapeutic agents in colon cancer cells. Clin Cancer Res 2000, 6(3):1198-1199.

10. Xiao H, Zhang Q, Lin Y, Reddy BS, Yang CS: Combination of atorvastatin and celecoxib synergistically induces cell cycle arrest and apoptosis in colon cancer cells. Int J Cancer 2008, 122(9):2115-2124.

11. Berglund G, Elmstahl S, Janzon L, Larsson SA: The malmo diet and cancer study: design and feasibility. J Intern Med 1993, 233(1):45-51.

12. Brandstedt J, Wangefjord S, Nodin B, Gaber A, Manjer J, Jirstrom K: Gender, anthropometric factors and risk of colorectal cancer with particular reference to tumour location and TNM stage: a cohort study. Bio/ Sex Differ 2012, 3(1):23.

13. Brandstedt J, Wangefjord S, Borgquist S, Nodin B, Eberhard J, Manjer J, Jirstrom K: Influence of anthropometric factors on tumour biological characteristics of colorectal cancer in men and women: a cohort study. J Transl Med 2013, 11:293.

14. Edge SB, Compton CC: The American joint committee on cancer: the 7th edition of the AJCC cancer staging manual and the future of TNM. Ann Surg Oncol 2010, 17(6):1471-1474.

15. Wangefjord S, Brandstedt J, Lindquist KE, Nodin B, Jirstrom K, Eberhard J: Associations of beta-catenin alterations and MSI screening status with expression of key cell cycle regulating proteins and survival from colorectal cancer. Diagn Pathol 2013, 8:10.

16. Larsson A, Johansson ME, Wangefjord S, Gaber A, Nodin B, Kucharzewska P, Welinder C, Belting M, Eberhard J, Johnsson A, Uhlen M, Jirstrom K: Overexpression of podocalyxin-like protein is an independent factor of poor prognosis in colorectal cancer. Br J Cancer 2011, 105(5):666-672.

17. Wangefjord S, Manjer J, Gaber A, Nodin B, Eberhard J, Jirstrom K: Cyclin D1 expression in colorectal cancer is a favourable prognostic factor in men but not women in a prospective, population-based cohort study. Bio/ Sex Differ 2011, 2(1):10.

18. Eberhard J, Gaber A, Wangefjord S, Nodin B, Uhlen M, Ericson Lindquist K, Jirstrom $\mathrm{K}$ : A cohort study of the prognostic and treatment predictive value of SATB2 expression in colorectal cancer. Br J Cancer 2012, 106(5):931-938.

19. Nodin B, Johannesson H, Wangefjord S, O'Connor DP, Ericson-Lindquist $K$, Uhlen M, Jirstrom K, Eberhard J: Molecular correlates and prognostic 
significance of SATB1 expression in colorectal cancer. Diagn Pathol 2012, 7(1):115.

20. Borgquist S, Jogi A, Ponten F, Ryden L, Brennan DJ, Jirstrom K: Prognostic impact of tumour-specific HMG-CoA reductase expression in primary breast cancer. Breast Cancer Res 2008, 10(5):R79.

21. Wangefjord S, Sundstrom M, Zendehrokh N, Lindquist KE, Nodin B, Jirstrom K, Eberhard J: Sex differences in the prognostic significance of KRAS codons 12 and 13, and BRAF mutations in colorectal cancer: a cohort study. Biol Sex Differ 2013, 4(1):17.

22. Nodin B, Zendehrokh N, Sundstrom M, Jirstrom K: Clinicopathological correlates and prognostic significance of KRAS mutation status in a pooled prospective cohort of epithelial ovarian cancer. Diagn Pathol 2013, 8:106.

23. Cheng $H$, Liang $H$, Qin Y, Liu Y: Nuclear beta-catenin overexpression in metastatic sentinel lymph node is associated with synchronous liver metastasis in colorectal cancer. Diagn Pathol 2011, 6:109.

24. Larsson AH, Nodin B, Syk I, Palmquist I, Uhlen M, Eberhard J, Jirstrom K: Podocalyxin-like protein expression in primary colorectal cancer and synchronous lymph node metastases. Diagn Pathol 2013, 8(1):109.

25. Barratt PL, Seymour MT, Stenning SP, Georgiades I, Walker C, Birbeck K, Quirke P: DNA markers predicting benefit from adjuvant fluorouracil in patients with colon cancer: a molecular study. Lancet 2002, 360 (9343):1381-1391.

26. Ribic CM, Sargent DJ, Moore MJ, Thibodeau SN, French AJ, Goldberg RM, Hamilton SR, Laurent-Puig P, Gryfe R, Shepherd LE, Tu D, Redston M, Gallinger S: Tumor microsatellite-instability status as a predictor of benefit from fluorouracil-based adjuvant chemotherapy for colon cancer. N Engl J Med 2003, 349(3):247-257.

27. Kim GP, Colangelo LH, Wieand HS, Paik S, Kirsch IR, Wolmark N, Allegra CJ: Prognostic and predictive roles of high-degree microsatellite instability in colon cancer: a National Cancer Institute-National surgical adjuvant breast and bowel project collaborative study. J Clin Oncol 2007, 25(7):767-772

28. Gaber A, Stene C, Hotakainen K, Nodin B, Palmquist I, Bjartell A, Stenman UH, Jeppsson B, Johnson LB, Jirstrom K: Effects of radiation therapy on tissue and serum concentrations of tumour associated trypsin inhibitor and their prognostic significance in rectal cancer patients. Radiat Oncol 2011, 6:100

29. Goldstein JL, Brown MS: Regulation of the mevalonate pathway. Nature 1990, 343(6257):425-430.

doi:10.1186/1746-1596-9-78

Cite this article as: Bengtsson et al.: HMG-CoA reductase expression in primary colorectal cancer correlates with favourable clinicopathological characteristics and an improved clinical outcome. Diagnostic Pathology 2014 9:78.

\section{Submit your next manuscript to BioMed Central and take full advantage of:}

- Convenient online submission

- Thorough peer review

- No space constraints or color figure charges

- Immediate publication on acceptance

- Inclusion in PubMed, CAS, Scopus and Google Scholar

- Research which is freely available for redistribution 